\title{
La tarjeta postal en España: usos y tendencias
}

\author{
Mariana LÓPEZ HURTADO \\ Centro de Estudios Políticos y Constitucionales \\ m.lopezhurtado@gmail.com
}

Recibido: Julio 2013

Aceptado: Septiembre 2013

Resumen: Desde el nacimiento de la tarjeta postal en 1869 los usos que se le han atribuido han ido variando con el paso del tiempo llegando a consolidar una importantísima industria editorial con gran popularidad entre los ciudadanos. Sin embargo, no existe ningún estudio que muestre el uso actual que tienen estos documentos en España y que permita conocer la valoración y la opinión que de ellos tienen los españoles. Por este motivo se presenta un estudio objetivo en el que se esclarecen estos aspectos y permite vislumbrar una valoración de la tarjeta postal resaltando su valor como medio de comunicación y documento coleccionable.

Palabras clave: Tarjeta postal; Tarjeta postal electrónica; Estudios de usuarios; Metodología de investigación; España

The postcard in Spain: uses and trends

\begin{abstract}
Since the birth of the postcard in 1869 the uses that have been attributed to it have varied over time, reaching to consolidate an important publishing industry and a great popularity among citizens. However, there is not any study that show the current use that these documents have in Spain and that show the appreciation and opinion that Spanish have of them. For this reason an objective study is presented in which these issues are clarified and glimpse an assessment of the postcard highlighting its value as a mean of communication and as a collectible document.
\end{abstract}

Keywords: Postcard; E-postcard; User studies; Research methology; Spain

\section{INTRODUCCIÓN}

Desde que en 1869 naciera en Austria la primera tarjeta postal como un producto pensado para el comercio cuyo fin principal era abaratar los costes en el correo postal, los usos que se le han ido atribuyendo han ido variando y ampliándose a lo largo de los años. Gracias a sus reducidas dimensiones, el coste de su franqueo en estos primeros años de vida resultaba bastante más económico que una carta ordinaria de las de la época. Por este motivo rápidamente estas tarjetas, también llamadas tarjetas de correspondencia, cobran popularidad y en tan solo un mes desde su aparición se venden en Austria cerca de un millón y medio de ejemplares. A 
partir de ese momento, su uso se generaliza enormemente llegando a consolidar una industria artística-editorial muy importante con enormes beneficios.

España tarda un poco más en sumarse al uso de estos documentos, y no será hasta 1871 cuando la Administración inste a la Fábrica del Sello a confeccionar la primera tarjeta postal. Sin embargo hasta 1873 no circula en España la primera tarjeta postal oficial. Estos dos años de retraso originan que una industria privada floreciente se lance a la edición de postales.

La explosión de la tarjeta postal ilustrada, y más concretamente de la postal fotográfica, viene de la mano de la apuesta que algunos grandes fotógrafos e industrias de la imagen hacen por estas tarjetas hacia finales del siglo XIX, cuando el progreso tecnológico permite que la imprenta reproduzca adecuadamente las fotografías y con ellas, tal y como afirma Bernardo Riego (1997: 34), se introduzcan grandes dosis de realismo gracias a la instantaneidad. Sin extendernos demasiado, nos gustaría resaltar el trabajo de algunos de estos fotógrafos que dedicaron tiempo y recursos a la tarjeta postal.

En primer lugar debemos destacar el trabajo realizado por la casa Hauser y Menet, la fototipia más prolífica e importante de todas las establecidas en España, con una producción de medio millón de tarjetas postales al mes a principios del siglo XX (Teixidor Cadenas, 1999: 9-23). Por su parte Josep Thomas en Barcelona, con un amplio equipo de fotógrafos repartidos por toda España, llega a ofrecer más de 20000 temas distintos.

En Madrid Antonio Cánovas del Castillo, Kaulak, gran aficionado al coleccionismo de postales, se dedica a la edición de tarjetas destacando de entre sus series alfabéticas, la serie «L» $\mathrm{y}$ «LL» o «iQuién supiera escribir!» con las Doloras de Campoamor de las que llega a vender 180000 ejemplares. También es de destacar el trabajo de Laurent, que fue aprovechado por sus sucesores para abrir una fototipia en 1889 cambiando la firma a «Sucesores de Laurent». En 1902 Lacoste se convierte en propietario de la galería de Laurent y continúa con la edición de postales combinado varias firmas y respetando las obras de Laurent.

Una de las fototipias con mayor continuidad en el tiempo es la editorial de Manuel Arribas creada en 1905 que cierra sus puertas tras ochenta y siete años de activad. El fotógrafo Zerkowitz, cuyos primeros trabajos fotográficos como profesional fueron destinados a ilustrar una serie de tarjetas postales, realiza una importante campaña de difusión de postales durante los años veinte y treinta en catálogos.

Con el atractivo estético de la postal a partir de 1900 se socializa su uso y se convierte en un documento coleccionable e intercambiable, siendo frecuentes en las revistas especializadas anuncios ofreciendo la posibilidad de establecer contacto para intercambiar postales de distintos lugares, y de muy variadas temáticas.

Desde principios del siglo XX hasta finales de la I Guerra Mundial la tarjeta postal vive lo que algunos autores como Gaja i Colomer (2009) denominan la «edad de oro». A partir de ese momento su uso disminuye hasta que en los años 70 vuelve a cobrar gran popularidad y se convierten de nuevo en un fenómeno de 
masas, siendo muy utilizadas en los viajes turísticos como elemento de recuerdo o como muestra a familiares y amigos de los lugares que se han visitado.

A partir de los años 80 y 90 se produce de nuevo un descenso en el uso de las tarjetas postales, y a finales del siglo XX y durante el siglo XXI nacen las conocidas tarjetas postales electrónicas. Estas nuevas tarjetas pueden ser enviadas a través de Internet y permiten al emisor personalizar al máximo la imagen de la postal, pudiendo incluso adjuntar sonido o video. Sin embargo, y a pesar de la gran popularidad que estas pudieran haber cobrado al estar inmersas en las tecnologías de la información y la comunicación, su uso no se ha extendido tanto como cabría esperar a priori.

Actualmente no existen estudios que muestren la valoración de las tarjetas postales y que nos permitan conocer su verdadero uso, que analicen cómo ha afectado a la postal tradicional el nacimiento de la postal electrónica y con los que podamos establecer relaciones con distintas variables sociodemográficas. Por estos motivos es necesario llevar a cabo un estudio objetivo centrado en todas estas variables con el que poder esclarecer todos estos aspectos.

\section{OBJETIVOS}

Los objetivos de este estudio ya los hemos adelantado brevemente en la introducción pero, de forma más detallada, se centran en los siguientes aspectos:

- Conocer el porcentaje de ciudadanos españoles que adquieren, intercambian, envían o reciben postales.

- Detectar qué características de las tarjetas postales son más valoradas y cuáles menos.

- Averiguar el uso real que hacen los ciudadanos españoles de las tarjetas postales.

- Determinar si existen patrones de uso o preferencias en cuanto a las tarjetas postales en función de la edad, nivel de estudios, sexo y características similares de los encuestados.

- Conocer la valoración y uso de tarjetas postales electrónicas.

\section{METODOLOGÍA}

Con el fin de conocer el uso de las tarjetas postales en España realizamos un estudio de opinión a partir de una encuesta utilizando un muestreo de tipo probabilístico (Couper, 2000), que fue enviada por correo electrónico a una muestra de selección aleatoria. La encuesta también fue impresa, para ser distribuida en este soporte.

Según Abad García (2005: 65), un cuestionario es un instrumento de recogida de datos constituido por un conjunto de preguntas, diseñadas cuidadosamente, 
sobre los hechos y aspectos que interesan en una investigación. En función de esta definición elaboramos un cuestionario de treinta y tres preguntas con las características que detallamos a continuación.

Se trata de un cuestionario autoadministrado, puesto que los entrevistados leen las preguntas y las responden sin interactuar directamente con el investigador. Esto garantiza la objetividad, la rapidez, facilidad de administración, así como el bajo coste. Al mismo tiempo es un cuestionario semiestructurado que combina preguntas cerradas (20), abiertas (3) y semicerradas (10). Se permite seleccionar la opción N/A (No aplicable) en el caso de preguntas con presentación de matrices de escala numérica, o mediante la opción N. S. / N. C. / (No sabe / No contesta) en otro tipo de respuestas.

Es completamente anónimo y se divide en 5 áreas temáticas para analizar con precisión las respuestas de los encuestados:

1. Datos personales

2. Uso y distribución de las tarjetas postales

3. Envío y recepción de tarjetas postales

4. Actividad turística

5. Valoración global de la tarjeta postal

El cuestionario se prepara con la herramienta en línea Google Docs (actualmente renombrada como Google Drive), por tratarse de una plataforma gratuita y por sus posibilidades de gestión mediante una cuenta de correo Gmail asociada al propio cuestionario. A continuación se envía por correo electrónico y se distribuye en papel a un grupo de mil personas aleatorio, pidiendo su colaboración para distribuirlo entre otros usuarios. También se contacta con asociaciones de tarjetas postales, blogs especializados y varios grupos de Facebook para lograr la máxima difusión posible.

Al mismo tiempo se tienen en cuenta otros aspectos importantes a la hora de elaborar el cuestionario como son los siguientes.

\subsection{TIPO DE MUESTREO}

Con nuestra investigación pretendemos conocer la opinión de una muestra representativa de los ciudadanos españoles mayores de 18 años, sin embargo, no nos es posible entrevistar a toda la población por lo que decidimos emplear un tipo de muestreo de carácter probabilístico. La ventaja frente a otros, reside tal y como afirma Alaminos Chica y Castejón Costa (2006: 55) en poder estimar el grado de error que afecta a la medición de la muestra. 


\subsection{TAMAÑO DE LA MUESTRA}

Los sondeos de carácter político, según Arias López (2007), requieren de entre 500 y 1000 encuestados. En el caso de CIES $^{1}$, esta empresa selecciona una muestra representativa de entre 400 y 2000 entrevistados, para que la muestra sea fiable en sondeos electorales esta oscila entre los 500 y los 1000 entrevistados.

En cualquier caso, en general se utilizan unas expresiones para el cálculo de las muestras en función del tamaño de la población objeto de estudio y del error admisible en los resultados. En este caso estimaremos como válido un error inferior al 5\%. Así, aplicando la expresión común de cálculo del tamaño de la muestra (infinitas) definida a continuación (Grande; Abascal, 2005) obtenemos:

$$
\begin{aligned}
& n=\frac{N k^{2} P Q}{e^{2} N+k^{2} S^{2}} \text { lo que para poblaciones grandes es equivalente a: } \\
& n=\frac{k^{2} P Q}{e^{2}} \text { resultando en nuestro caso } \mathrm{n}=384
\end{aligned}
$$

Siendo: $\mathrm{n}$ el tamaño de la muestra; $\mathrm{N}$ el universo de estudio; $\mathrm{P}$ representa al porcentaje de la población que posee una característica; $\mathrm{Q}=1-\mathrm{P}$, para datos no segmentados, el caso más desfavorable es el de $\mathrm{P}=\mathrm{Q}=0,5$ por lo que tomaremos este valor; $\mathrm{k}$ un valor obtenido mediante niveles de confianza, generalmente se asigna un valor de 1,96 para un nivel de confianza del 95\%; y el error máximo admisible, en nuestro caso del 5\%.

Teniendo en cuenta estas referencias estableceremos como válida una muestra superior a 384 respuestas.

\subsection{TRATAMIENTO DE LA INFORMACIÓN}

Las respuestas de cada persona son anónimas. A pesar de que los cuestionarios contestados a través de correo electrónico permiten conocer ciertos datos personales de los encuestados, estos no figuran en el cuestionario, tampoco se guardan o almacenan sino que son eliminados una vez recibimos el cuestionario. De esta manera garantizamos el anonimato y a la vez evitamos que los encuestados se sientan cohibidos a la hora de responder a las preguntas planteadas.

\footnotetext{
${ }^{1}$ CIES se funda en el año 1981 y pertenece a The Research Alliance, cadena internacional de institutos de estudios de opinión y mercado. Cuenta con la certificación AENOR de empresa registrada con el número de registro ER-1528/2003. Es miembro de AEDEMO (Asociación Española de Estudios de Mercado y Opinión), ESOMAR (Asociación Europea de Investigación de Opinión y Mercados).
} 
Todas las preguntas aparecen ordenadas, de lo general a lo particular (técnica del embudo), para poder situar al usuario e ir avanzando en el nivel de especificidad del análisis.

\section{RESULTADOS}

El envío de 1000 encuestas comenzó el 23 de julio de 2011 y el cuestionario estuvo en circulación durante 10 meses hasta el 24 de mayo de 2012. El total de cuestionarios cumplimentados recibidos fue de 467 tanto de forma electrónica como, en algunos casos, en formato papel. No obstante, ha habido un reducido porcentaje de respuestas que no se han considerado válidas debido a que los cuestionarios estaban incompletos (tabla 1).

Tabla 1. Número total de respuestas

\begin{tabular}{|l|c|}
\hline & Total \\
\hline Enviados & 1000 \\
\hline Total de respuestas & 467 \\
\hline Respuestas erróneas & 5 \\
\hline Respuestas válidas & $\mathbf{4 6 2}$ \\
\hline
\end{tabular}

A partir de las respuestas obtenidas es posible calcular el error obtenido con una muestra de tamaño 462, siendo (Grande; Abascal, 2005):

$$
e=k \sqrt{\frac{P Q}{n}} \quad=0,0456 \text { o } 4,56 \%
$$

\subsection{DATOS PERSONALES}

Del total de encuestados la mayoría son mujeres, con un porcentaje del $68,18 \%$ frente al 31,82\% de hombres, es decir más del doble. En cuanto a la distribución de las encuestas por edades, se han establecido seis grupos, cuatro de ellos comprendidos entre los 18 y los 65 años, más dos en la franja superior e inferior a este rango. Sin embargo las escasas respuestas que han recibido estos dos últimos grupos han provocado que los resultados obtenidos nos impongan restricciones respecto a la validez de la realización de estimaciones fiables. En la franja de edades entre 18 y 65 años, se ha obtenido un porcentaje de respuestas de entre el 20 y el $30 \%$ del total. El resumen de las respuestas por rangos de edad ha sido:

- Menor de 18: 0,65\%

- Entre 18-30: 28,14\%

- Entre 31-40: 25,54\% 
- $\quad$ Entre 41-50: 21,86\%

- Entre 51-65: 21,00\%

- Mayor de 65: 2,81\%

Se ha tratado de distribuir el cuestionario entre personas con diferentes niveles de estudios para observar si existen variaciones con respecto al uso de tarjetas postales. Es evidente que el número de personas sin estudios de ningún tipo es prácticamente irrelevante $(0,87 \%)$, al igual que el de las personas que han cursado estudios diferentes a los propuestos por nosotros. Los estudios de licenciatura y asimilados se perfilan como los más habituales entre los encuestados con un $47,84 \%$, seguidos por los de diplomatura que representan el $16,23 \%$ de las respuestas totales. En resumen las respuestas obtenidas por niveles de estudios han sido:

- Sin estudios: $0,87 \%$

- Primarios: $7,79 \%$

- Secundarios: $4,76 \%$

- Bachillerato / FP: $13,85 \%$

- Diplomatura: $16,23 \%$

- Licenciatura: $47,84 \%$

- Doctorado: $8,23 \%$

- Otros: $0,43 \%$

También se han hecho distribuciones por regiones y por la ocupación actual de los encuestados pero no se han encontrado, en general, variaciones apreciables en las tendencias relacionadas con las tarjetas postales para las diferentes categorías observadas por lo que no serán tratadas en este trabajo.

\subsection{USO Y DISTRIBUCIÓN DE LAS TARJETAS POSTALES}

En este apartado se han analizado los aspectos relativos a la adquisición y a la valoración, en general, de las tarjetas postales. Respecto a este último aspecto, se ha consultado a los encuestados si consideran la tarjeta postal como un buen medio de comunicación ofreciendo tres posibles respuestas: sí, no y NS/NC (no sabe o no contesta). Los resultados de esta pregunta muestran una valoración muy positiva de las tarjetas postales como medio de comunicación con un $69,48 \%$ de respuestas afirmativas.

La adquisición de estos materiales a lo largo del tiempo ha sido otro de los aspectos evaluados. Lo que tratamos de determinar es si los encuestados han adquirido alguna vez tarjetas postales y poder caracterizarlos en función de los datos sociodemográficos (sexo, edad y estudios). Es relevante señalar que tan solo el $8,44 \%$ de los encuestados afirma que nunca ha adquirido una tarjeta postal.

Si contextualizamos estos mismos datos de adquisición en función del sexo de los encuestados, podemos apreciar que el $14,97 \%$ de los hombres nunca ha adquirido una tarjeta postal frente al 5,40\% de las mujeres. Es de destacar que esta 
diferencia de 10 puntos se mantendrá en diferentes aspectos relacionados con el uso de la postal de los estudiados en este trabajo.

Al mismo tiempo se ha tratado de determinar si existe alguna relación entre la adquisición de tarjetas postales y la edad de los encuestados. Constatamos que la relación entre las variables edad-adquisición de tarjetas postales (tabla 2) sigue una tendencia decreciente, es decir que los grupos de menor edad tienen un menor hábito de adquisición de tarjetas postales que los grupos de mayor edad. Aunque también hay una pequeña disfunción en el grupo de 51-65 años cuyos encuestados han respondido que adquieren o han adquirido tarjetas postales $(91,75 \%)$ en un porcentaje inferior al grupo de edad que le precede $(95,05 \%)$.

Tabla 2. Adquisición de tarjetas postales en función de la edad

\begin{tabular}{|l|c|c|}
\hline & Sí adquiere o ha adquirido & Nunca ha adquirido \\
\hline Menor de 18 & $66,67 \%$ & $33,33 \%$ \\
\hline $\mathbf{1 8 - 3 0}$ & $86,15 \%$ & $13,85 \%$ \\
\hline $\mathbf{3 1 - 4 0}$ & $94,07 \%$ & $5,93 \%$ \\
\hline $\mathbf{4 1 - 5 0}$ & $95,05 \%$ & $4,95 \%$ \\
\hline $\mathbf{5 1 - 6 5}$ & $91,75 \%$ & $8,25 \%$ \\
\hline Mayor de 65 & $100,00 \%$ & $0,00 \%$ \\
\hline
\end{tabular}

Igualmente se ha comparado la adquisición de tarjetas postales a lo largo del tiempo con el nivel de estudios (tabla 3). El 95,02\% de los licenciados afirma haber adquirido alguna vez tarjetas postales frente al $80,56 \%$ y $75 ; 00 \%$ de los encuestados con estudios primarios y sin estudios respectivamente. Así, se aprecia una relación descendente en cuanto a la adquisición de tarjetas postales en función del nivel de estudios, esto es, a mayor nivel de estudios, menor porcentaje de personas que nunca han adquirido tarjetas postales.

Tabla 3. Adquisición de tarjetas postales en función del nivel de estudios

\begin{tabular}{|l|c|c|}
\hline & Sí adquiere o ha adquirido & Nunca ha adquirido \\
\hline Sin estudios & $75,00 \%$ & $25,00 \%$ \\
\hline Primarios & $80,56 \%$ & $19,44 \%$ \\
\hline Secundarios & $90,91 \%$ & $9,09 \%$ \\
\hline Bachillerato/ FP & $87,50 \%$ & $12,50 \%$ \\
\hline Diplomatura & $90,67 \%$ & $9,33 \%$ \\
\hline Licenciatura & $95,02 \%$ & $4,98 \%$ \\
\hline Doctorado & $92,11 \%$ & $7,89 \%$ \\
\hline Otros & $100,00 \%$ & $0,00 \%$ \\
\hline
\end{tabular}


Por otra parte se ha consultado acerca de la finalidad con la que se adquieren tarjetas postales. Se han recogido 422 respuestas a la pregunta que proporcionan un total de 557 motivos de adquisición ya que la pregunta es de respuesta múltiple. El principal motivo de adquisición de tarjetas postales es el envío con un $63,20 \%$ de las respuestas. Nada desdeñable es el motivo «coleccionismo» que representa un $34,65 \%$ y entre otros motivos $(2,15 \%$ del total de respuestas) se encuentra la opinión de usuarios que resaltan su finalidad como el recuerdo, su valor como buena fotografía o para recordar celebraciones.

Por último, en este apartado se ha solicitado a los encuestados que valoren el precio medio actual de las tarjetas postales que se encuentra en torno a los 60 céntimos de euro. Para realizar esta medición los encuestados han asignado una cifra a su valoración en una escala numérica del 1 al 5, en la que el 1 representa que el precio es muy bajo y el 5 que es muy elevado. La valoración media resultante fue de 2,94, lo que implica una consideración de la tarjeta postal como un bien ni muy caro ni excesivamente barato.

\subsection{ENVÍO Y RECEPCIÓN DE TARJETAS POSTALES}

Este apartado identifica todos los aspectos relacionados con el envío de tarjetas postales y las preferencias de los encuestados al respecto. También se trata de determinar si existen tendencias diferenciales entre los factores sociodemográficos de los encuestados y los aspectos relacionados con el envío de tarjetas postales estudiados.

Una de las preguntas más destacadas de este apartado pretende determinar qué porcentaje de encuestados no envía actualmente tarjetas postales y las razones para no hacerlo. En total un 23,38\% afirma no utilizar este medio actualmente y entre las principales razones que alegan se encuentran:

- Falta de tiempo: $19,12 \%$

- Falta de privacidad al ir la postal sin sobre: $13,97 \%$

- Pereza: $48,53 \%$

- Coste elevado: $2,21 \%$

- Otros motivos: $16,18 \%$

Dentro de la categoría «Otros motivos» se ha permitido definir con mayor precisión estas causas, así, entre las consignadas, destaca la preferencia de otros medios de comunicación $(9,56 \%)$ o el retraso del correo ordinario $(1,74 \%)$ en un momento en el que la inmediatez de las comunicaciones es un elemento altamente demandado por la sociedad.

El uso actual de tarjetas postales también se ha caracterizado en función de las tres variables sociodemográficas definidas anteriormente. Si nos centramos en el sexo de los encuestados, observamos que el $80,63 \%$ de las mujeres afirman enviar tarjetas postales actualmente frente a un $68,03 \%$ de hombres que también lo hace. Es curioso destacar que los rangos de edades intermedias (31-40 y 41-50) son los que a 
día de hoy más utilizan las tarjetas postales con un $84,75 \%$ y un $78,22 \%$, frente a los más jóvenes y a los más ancianos con un $66,67 \%$ y un $61,54 \%$ respectivamente.

Existen variaciones más destacadas en lo que se refiere a los niveles de estudio. De nuevo las personas que mayor uso hacen actualmente de las postales son las que poseen estudios superiores: doctorado $(81,58 \%)$, licenciatura $(80,09 \%)$ y diplomatura $(82,67 \%)$. En total destaca que el $81,45 \%$ de los encuestados con estudios superiores utilicen actualmente la tarjeta postal frente al $63,80 \%$ con estudios medios de lo que subyace la buena valoración del soporte.

El coleccionismo de tarjetas postales es un fenómeno importantísimo históricamente ya que con él aumenta el valor de la tarjeta postal y hace que se genere una actividad muy importante de intercambio entre coleccionistas. Ahora bien, en este caso entendemos coleccionismo en el sentido más amplio del término, es decir no solamente incluimos a aquellas personas interesadas en la adquisición de todo tipo de postales sino también a aquellas que compran tarjetas postales de los sitios que han visitado para conservarlas como recuerdo o almacenar las que envían familiares y amigos. En función de este criterio el $77,33 \%$ de los encuestados afirma coleccionar estos documentos. Los motivos para coleccionar tarjetas postales son de muy variada naturaleza, destacando el valor artístico por encima de otros con un 33,14\%. Cabe mencionar que prácticamente ningún encuestado ha aludido al valor económico de las postales $(0,34 \%)$ o a su valor en cuanto al volumen de tarjetas $(0,00 \%)$ como motivo para coleccionarlas. A continuación mostramos un resumen de estos motivos:

- Valor artístico: 33,14\%

- Valor en cuanto a la cantidad: $0,00 \%$

- Valor como recuerdo: $23,46 \%$

- Valor económico: 0,34\%

- Valor fotográfico: $13,90 \%$

- Valor histórico: 6,49\%

El soporte de la tarjeta postal es uno de los aspectos más importantes e interesantes del uso de la postal, sobre todo teniendo en cuenta la aparición relativamente reciente de los nuevos medios digitales. Por este motivo hemos consultado a los encuestados acerca de sus preferencias en cuanto al soporte de la tarjeta postal tanto para el envío como para la recepción de las mismas. Los resultados son sorprendentes pues, tanto para el envío como para la recepción el soporte papel-cartulina es el preferido por los encuestados. Como decimos es sorprendente teniendo en cuenta el gran uso que tienen las tecnologías de la información y la comunicación actualmente. De ahí que consideráramos previamente que, al menos para el envío las tarjetas electrónicas, tuvieran mayor incidencia. Sin embargo, los resultados muestran que tan solo el $4,11 \%$ prefiere enviar tarjetas postales electrónicas, frente al 64,29\% que prefiere el soporte tradicional (figura 1). En la recepción esta diferencia es aún mayor, el 73,81\% 
prefiere el soporte papel-cartulina frente al 3,90\% que las prefiere electrónicas (figura 2).

Figura 1. Preferencias en cuanto al soporte de la tarjeta postal en el envío

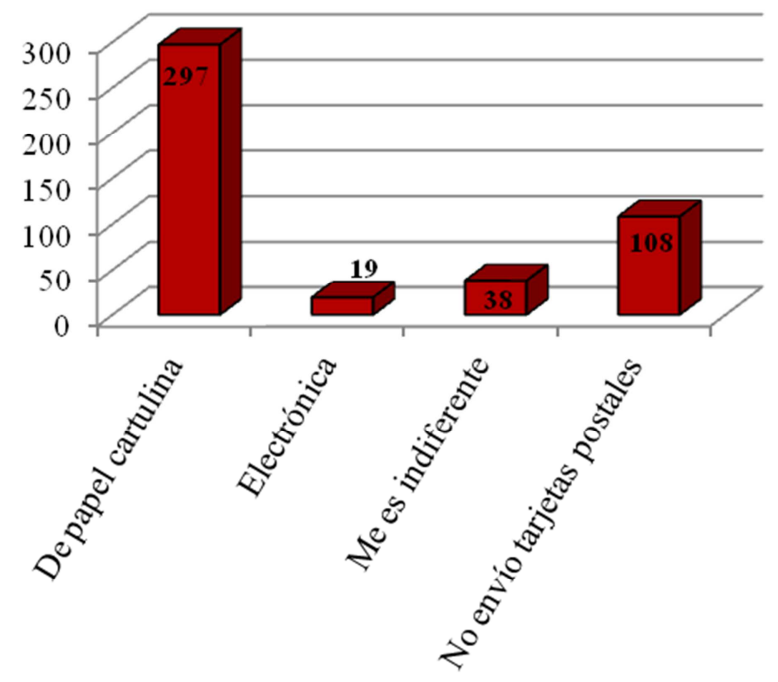

$\square$ Respuestas

Figura 2. Preferencias en cuanto al soporte de la tarjeta postal en la recepción

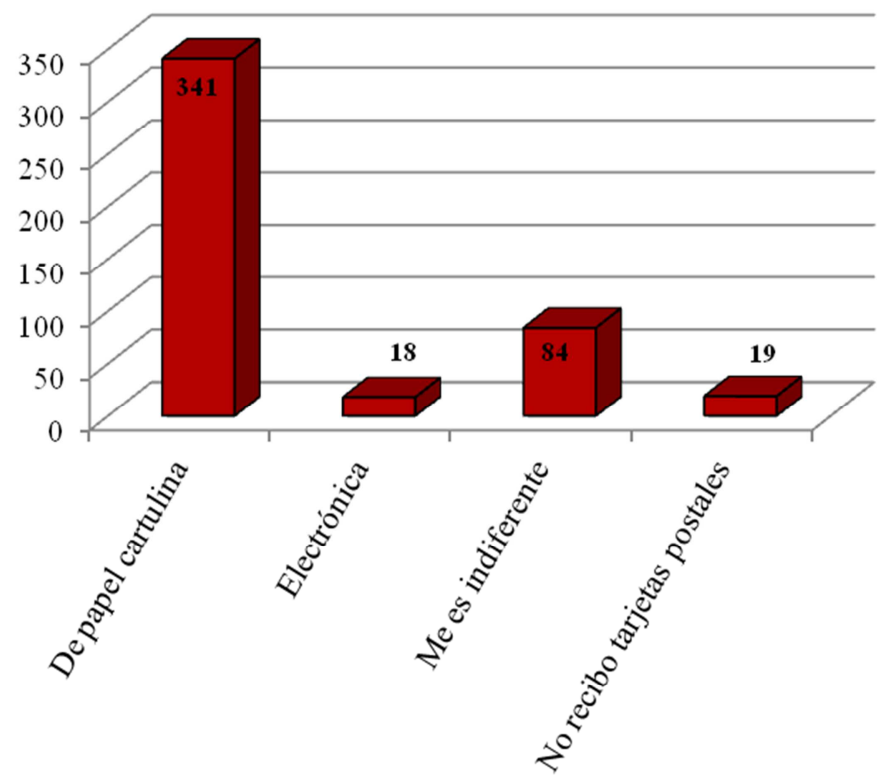

口Respuestas 
Finalmente se ha analizado el uso de las tarjetas postales electrónicas y se ha constatado que siguen siendo minoritarias con un $29,65 \%$ de usuarios que las utilizan. Al igual que las postales en soporte tradicional, las electrónicas son mayoritariamente utilizadas por mujeres con un 33,33\% frente al $21,77 \%$ de los hombres. En cuanto a la edad, entre los grupos intermedios (31-65 años) el uso de tarjetas postales electrónicas no muestra diferencias significativas: en torno a un $30 \%$. Sin embargo, el grupo de mayores de 65 realizan un uso residual de este medio con un 7,69\%. También es destacable que ninguno de los encuestados menores de 18 años afirme usar tarjetas postales electrónicas aunque, como hemos dicho al principio, el reducido volumen de respuestas de este rango de edad hace que estos datos no sean concluyentes.

\subsection{ACTIVIDAD TURÍSTICA}

La estrecha relación que guarda el envío de tarjetas postales con la realización de viajes es el aspecto que trata de estudiarse en este apartado. Hemos tratado de establecer correspondencias entre periodos en la realización de viajes, preferencias de destinos turísticos y uso de la tarjeta postal en los viajes frente a variables ya obtenidas en apartados anteriores.

Para conseguirlo hemos realizado diferentes preguntas relativas a los periodos del año en los que se realizan los viajes para comprobar si existen relaciones en las tendencias del envío de tarjetas postales. Se han establecido cinco periodos: verano, navidades, Semana Santa, otros periodos y resto del año. En la mayoría de los periodos no parece haber grandes diferencias, es decir entre el 70 y el 85 por ciento de los encuestados envían tarjetas postales. Sin embargo quienes realizan sus viajes en Semana Santa y, sobre todo, aquellos que los realizan en Navidad, no suelen enviar tarjetas postales. La siguiente tabla muestra un resumen de las respuestas (tabla 4):

Tabla 4. Envío de tarjetas postales en función del periodo vacacional

\begin{tabular}{|c|c|c|c|c|c|c|c|c|c|c|}
\hline \multirow[b]{3}{*}{ Opciones } & \multicolumn{10}{|c|}{ Época del año preferente para realizar viajes } \\
\hline & \multicolumn{2}{|c|}{ Verano } & \multicolumn{2}{|c|}{ Navidades } & \multicolumn{2}{|c|}{$\begin{array}{c}\text { Semana } \\
\text { Santa }\end{array}$} & \multicolumn{2}{|c|}{$\begin{array}{c}\text { Otros } \\
\text { periodos }\end{array}$} & \multicolumn{2}{|c|}{$\begin{array}{l}\text { Resto del } \\
\text { año }\end{array}$} \\
\hline & Resp. & $\%$ & Resp. & $\%$ & Resp. & $\%$ & Resp. & $\%$ & Resp. & $\%$ \\
\hline $\begin{array}{l}\text { Sí envía actualmente } \\
\text { tarjetas postales }\end{array}$ & 204 & 73,91 & 2 & 40,00 & 10 & 55,56 & 64 & 83,12 & 71 & 85,54 \\
\hline $\begin{array}{l}\text { No envía actualmente } \\
\text { tarjetas postales }\end{array}$ & 72 & 26,09 & 3 & 60,00 & 8 & 44,44 & 13 & 16,88 & 12 & 14,46 \\
\hline Total & 276 & 100,00 & 5 & 100,0 & 18 & 100,00 & 77 & 100,00 & 83 & 100,00 \\
\hline
\end{tabular}

También se ha consultado si los principales destinos de los viajes se encontraban en España o en el extranjero y se ha tratado de determinar si estos 
destinos afectaban al envío actual de tarjetas. Sin embargo, hemos obtenido que tanto los encuestados cuyos destinos principales se encuentran en España como aquellos que suelen viajar al extranjero realizan un uso prácticamente idéntico de la postal con un $74,83 \%$ y $79,39 \%$ respectivamente.

Seguidamente se ha propuesto a los encuestados una serie de destinos turísticos para que sean valorados mediante un criterio numérico del 1 al 5 correspondiendo el 1 a una puntuación otorgada al destino muy negativa y el 5 muy positiva. Los destinos propuestos han sido: playa, montaña y espacios naturales, turismo cultural, turismo gastronómico y cruceros. El objetivo era comprobar si aquellas personas que valoraban con mayor puntuación un destino turístico hacían mayor uso de las tarjetas postales, es decir, caracterizar a los destinos turísticos con los usuarios de las tarjetas postales y, al mismo tiempo, determinar si los encuestados que prefieren un determinado destino turístico tienen mayor afición a enviar postales.

De este modo, hemos determinado que en la mayoría de los destinos no hay diferencias sustanciales entre las diferentes valoraciones del propio destino realizadas por los encuestados y el uso más alto o más reducido de la tarjeta postal. Este hecho ha sido observado en los destinos playa (valoración media 3,23), montaña y espacios naturales (valoración media 3,50), turismo gastronómico (valoración media 2,68) y cruceros (valoración media 1,79). En cambio, en el turismo cultural, con una valoración media de 4,03 sobre 5 (figura 3), hemos encontrado que existe una correspondencia directa entre las valoraciones más altas y el uso de la postal, lo que podría determinar que los encuestados que frecuentan estos destinos turísticos realizan un mayor uso de las tarjetas postales. Los encuestados que mejor valoran este tipo de turismo son los que actualmente mayor uso hacen de la tarjeta postal.

Figura 3. Relación entre el envío de tarjetas postales y la valoración del 1 al 5 del destino turístico «turismo cultural»

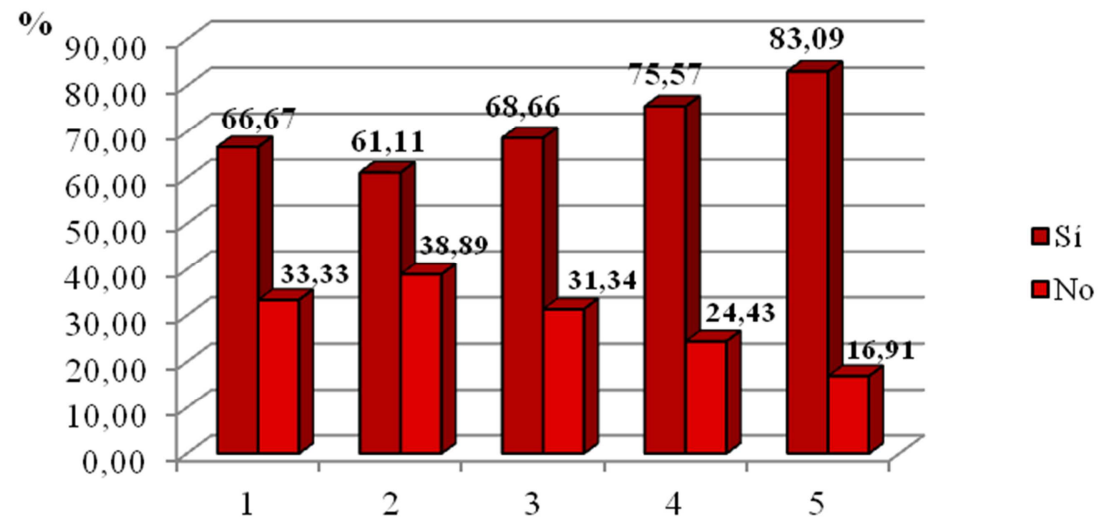

Por otra parte hemos consultado si los encuestados envían actualmente tarjetas postales durante sus viajes y en caso de no hacerlo sus principales motivos. Hemos 
detectado que el $46,75 \%$ no envía tarjetas postales durante sus viajes y los principales motivos para no hacerlo son: pereza $(60,31 \%)$, falta de tiempo $(22,52 \%)$, falta de privacidad al ir la postal sin sobre $(8,40 \%)$ y, en menor medida, el coste elevado de este medio (1,15\%).

\subsection{VALORACIÓN GLOBAL DE LA TARJETA POSTAL}

En este apartado se recogen las valoraciones personales de los encuestados a modo de conclusión y con el fin de abrirles la posibilidad de expresar aspectos que no han tenido cabida en el resto de bloques de la encuesta. En general se trata de preguntas abiertas en las que se les propone a los encuestados un tema o una frase para que sea completada por ellos. Por el interés de las respuestas ofrecidas hemos seleccionado una pregunta en la que proponíamos la frase: «Las tarjetas postales me parecen...» (tabla 5).

En función de las respuestas obtenidas hemos realizado una recopilación de los temas más recurrentes. Puesto que se trata de una pregunta abierta, de respuesta libre, ha sido laborioso poder aunar respuestas por su enorme variedad. En total se han identificado 514 valoraciones diferentes correspondientes a respuestas con múltiples opciones que ofrecen distintas características o peculiaridades de las tarjetas postales.

Las más destacadas corresponden a su valor como recuerdo, siendo definidas como elementos evocadores del tiempo perdido y que representan para un porcentaje elevado de los encuestados $(21,01 \%)$ un reflejo perfecto del lugar donde han estado y que les permite revivir con nostalgia $(0,58 \%)$ recuerdos gracias a las imágenes y a los textos que contiene. El valor como detalle para con la gente conocida o que otras personas tienen con los encuestados es muy importante $(16,73 \%)$. Los principales motivos relacionados con este aspecto definen a la tarjeta postal como una forma rápida $\mathrm{y}$ elegante de demostrar afecto o a personas significativas, pero a la vez una forma sencilla y cariñosa de enviar recuerdos desde otro lugar. Se trata de un medio más cálido que los modernos medios tecnológicos.

Su valor como medio de comunicación y su capacidad de acercar personas y de comunicar las experiencias de un viaje son algunas de las características resaltadas por los encuestados $(10,89 \%)$, en este sentido son un excepcional documento para mostrar a otros el lugar en el que se encuentran y que, al mismo tiempo, sirve de elemento publicitario del lugar visitado $(1,36 \%)$. Entre quienes destacan su valor como medio de comunicación también hay quien señala que su uso es anecdótico $(0,58 \%)$. Algunos ya las definen como medio alternativo $\mathrm{u}$ original de comunicación $(0,78 \%)$, aún teniendo presente que cuentan con siglo y medio de historia a sus espaldas. Pero también hay una parte importante que señala que se trata de objetos de otro tiempo, anticuadas, irrelevantes, innecesarias, desfasadas, pasadas de moda $(7,98 \%)$ o incluso anacrónicas $(0,39 \%)$. Hay quienes señalan que esto se debe a que han sido desplazadas por la fotografía digital y que, en cierto 
modo la idea de inmediatez que se ha impuesto en la sociedad actual hace difícil su convivencia con los medios tecnológicos $(0,97 \%)$ en comparación con la lentitud del correo tradicional $(1,36 \%)$.

Pero al mismo tiempo, otros señalan que el envío de postales es una tradición que merece la pena conservar $(2,92 \%)$ por su carácter excepcional y cercano de comunicación y que es, a la vez, una forma de preservar la cultura que no debería perderse e incluso definen las tarjetas como necesarias. Algunos de los encuestados que opinan que es una tradición a conservar y aluden a ellas como objetos en vías de extinción o ya desaparecidos y se refieren a la tarjeta postal como una costumbre o tradición a recuperar $(0,78 \%)$.

Existen otras opciones minoritarias que definen las tarjetas postales como divertidas $(2,53 \%)$, bonitas, emotivas o entrañables $(13,81 \%)$ e incluso románticas $(0,97 \%)$, como un fantástico medio de felicitación especialmente en ocasiones especiales $(2,72 \%)$, un simple souvenir $(0,97 \%)$, interesantes $(2,53 \%)$, útiles y prácticas $(0,78 \%)$, valiosas $(0,39 \%)$, una curiosidad $(1,36)$, o que señalan la poca privacidad del soporte al enviarse normalmente sin sobre $(0,39 \%)$ o quienes piensan que son un poco caras $(0,39 \%)$.

$\mathrm{Su}$ valor como objeto de colección es otro de los aspectos destacables $(3,89 \%)$ haciendo hincapié que esto solo sirve para las tarjetas en papel porque las electrónicas son más difíciles de conservar. También hay encuestados que las definen como antigüedades para coleccionistas $(0,39 \%)$. Por último destaca el valor documental de la tarjeta postal, por el tema que aquí nos ocupa, es uno de los aspectos señalados por los encuestados $(4,28 \%)$. Las tarjetas postales son, como documento, testigos del tiempo y de los lugares que representan y son testimonios escritos de los remitentes. Pero además se trata de documentos para la memoria colectiva, una verdadera etapa de la historia de la fotografía, elementos constitutivos del patrimonio cultural por su valor estético e histórico como reflejo de la sociedad que representan. Algunos de los encuestados que destacan la utilidad documental de la tarjeta postal se refieren también a su valor etnográfico $(0,58 \%)$.

Tabla 5. Resumen de respuestas a la pregunta «Las tarjetas postales me parecen...»

\begin{tabular}{|l|c|c|}
\hline \multicolumn{1}{|c|}{ Opciones } & Respuestas & $\mathbf{\%}$ \\
\hline Recuerdo & 108 & 21,01 \\
\hline Detalle & 86 & 16,73 \\
\hline Valor documental & 22 & 4,28 \\
\hline Felicitación & 14 & 2,72 \\
\hline Medio de comunicación & 56 & 10,89 \\
\hline Forma original de comunicación & 4 & 0,78 \\
\hline No deben perderse & 15 & 2,92 \\
\hline Bonitas & 71 & 13,81 \\
\hline
\end{tabular}




\begin{tabular}{|l|c|c|}
\hline Obsoletas, irrelevantes, anticuadas & 41 & 7,98 \\
\hline Publicitario del lugar & 7 & 1,36 \\
\hline Valiosas & 2 & 0,39 \\
\hline Objeto de colección & 20 & 3,89 \\
\hline Lentas & 7 & 1,36 \\
\hline Interesantes & 13 & 2,53 \\
\hline Prácticas & 4 & 0,78 \\
\hline Poca privacidad & 2 & 0,39 \\
\hline Un poco caras & 2 & 0,39 \\
\hline Románticas & 5 & 0,97 \\
\hline Una curiosidad & 7 & 1,36 \\
\hline Anacrónicas & 2 & 0,39 \\
\hline Nostálgicas & 3 & 0,58 \\
\hline Un souvenir & 5 & 0,97 \\
\hline Divertidas & 13 & 2,53 \\
\hline Están desplazadas por otros medios & 5 & 0,97 \\
\hline Total & $\mathbf{5 1 4}$ & $\mathbf{1 0 0}$ \\
\hline
\end{tabular}

\section{CONCLUSIONES}

- La tarjeta postal es considerada un buen medio de comunicación por un $69,48 \%$ de los encuestados, a pesar de que entra en competencia directa con las tecnologías de la información y de las comunicaciones.

- Se constata que existe un bajo porcentaje de personas que nunca han adquirido una tarjeta postal $(8,49 \%)$ siendo aún menor el porcentaje en mujeres $(5,40 \%)$ que en hombres $(14,97 \%)$.

- Existe una tendencia creciente entre las personas que nunca han adquirido tarjetas postales según su edad y nivel de estudios. Es decir a menor edad de los encuestados el porcentaje de personas que nunca ha adquirido postales es creciente. Igualmente ocurre con los niveles de estudios más bajos.

- Un $23,38 \%$ de los encuestados no envía actualmente tarjetas postales siendo la pereza el principal motivo $(48,53 \%)$.

- $\quad$ El 77,33\% de los encuestados afirma coleccionar tarjetas postales aunque hay que valorar el término «coleccionar» en el sentido más amplio.

- El soporte papel-cartulina es el preferido tanto para el envío como para la recepción de tarjetas postales muy por encima de las postales electrónicas.

- No han podido extraerse grandes relaciones entre turismo y uso y envío de tarjetas postales. 
- El 46,75\% de los encuestados no envía postales actualmente durante sus viajes lo que supone un incremento considerable respecto del porcentaje de personas que no usan este soporte actualmente $(23,38 \%)$.

- Los usuarios destacan en las apreciaciones finales el valor de la tarjeta postal como recuerdo y recalcan que no desean que su uso decaiga o incluso que puedan llegar a desaparecer. Sin embargo la gran mayoría afirma no enviarlas por pereza.

\section{REFERENCIAS BIBLIOGRÁFICAS}

ABAD GARCÍA, M. F. (2005). Evaluación de la calidad de los sistemas de información. Madrid: Síntesis.

ALAMINOS CHICA, A. y CASTEJÓN COSTA, J. L. (2006). Elaboración, análisis e interpretación de encuestas, cuestionarios y escalas de opinión. Alcoy: Marfil; Alicante: Vicerrectorado de Calidad y Armonización Europea, Universidad de Alicante.

ARIAS LÓPEZ, J. (2007). ¿Qué son los estudios de opinión? $<$ http://sociologiaext.wordpress.com/2007/09/25/\%C2\%BFque-son-los-estudiosde-opinio-un-extracto-elaborado-por-jorge-arias-lopez/>. [Consulta: 04/05/2013].

COUPER, M. (2000). "Web Surveys a review of Issues and Approaches". Public Opinion Quarterly, v. 64, n. 4.

GAJA i COLOMER, X. (2009). Menuda història de la targeta postal a Manlleu 1903-2009. <http://www.cerclecartofilcatalunya.com/.../menuda-historiamanlleu.pdf $>$. [Consulta: 25/06/2013].

GRANDE, I.; ABASCAL, E. (2005). Análisis de encuestas. Madrid: ESIC.

RIEGO AMÉZAGA, B. [et al.] (1997). Santander en la tarjeta postal ilustrada (1897-1941): historia, coleccionismo y valor documental. Santander: Fundación Marcelino Botín.

TEIXIDOR CADENAS, C. (1999). La tarjeta postal en España 1892-1915. Madrid: Espasa Calpe. 